\title{
Aneurisma de aorta abdominal en paciente con situs inversus totalis
}

\author{
Abdominal aortic aneurysm in a patient with situs inversus totalis
}

Diego Téllez-Beltrán ${ }^{1}$, Alejandro González-Muñoz ${ }^{*}$, Vladimir Barón-Cifuentes ${ }^{3}$ y Juan M. Pradilla-Gómez ${ }^{4}$ ${ }^{1}$ Servicio de Cirugía General, Hospital Universitario San Ignacio, Pontificia Universidad Javeriana; ${ }^{2}$ Servicio de Cirugía General, Hospital Universitario Mayor Mederi, Universidad del Rosario; ${ }^{3}$ Servicio de Cirugia Vascular y Angiología, Hospital Universitario San Ignacio, Pontificia Universidad Javeriana; ${ }^{4}$ Facultad de Medicina, Pontificia Universidad Javeriana. Bogotá, Colombia

\section{Resumen}

Antecedentes: El situs inversus totalis (SIT) es una condición congénita infrecuente, en la cual los órganos asimétricos del abdomen y el tórax presentan una disposición en espejo; su asociación con aneurismas de aorta abdominal (AAA) es inusual y supone un reto quirúrgico. Caso clínico: Presentamos el caso de una paciente con SIT y AAA, por lo que se decide llevar a reparación quirúrgica. EI AAA en pacientes con SIT es infrecuente; en la literatura hay pocos casos reportados. $A$ pesar de ser una relación inusual, su tratamiento de forma electiva fue segura en nuestra paciente.

Palabras clave: Aneurisma. Situs inversus. Vascular.

\begin{abstract}
Background: The situs inversus totalis (SIT) is a rare congenital condition, in which the asymmetric organs of the abdomen and thorax have a mirror ubication; its association with abdominal aortic aneurysms, is unusual. Case report: We will present the case of a patient with SIT, who has an abdominal aortic aneurysm, taken to open surgical repair. The presentation of abdominal aortic aneurysms in patients with infrequent SIT, with few reported cases in the literature. Despite being an unusual relationship, elective treatment was safe, as we seen in our patient.
\end{abstract}

Key words: Aneurysm. Situs inversus. Vascular.

\section{Introducción}

La localización y la configuración de los órganos asimétricos en el cuerpo humano, como el hígado, el bazo, el corazón, los pulmones y el sistema gastrointestinal, se denomina situs; el situs solitus corresponde a la disposición de los órganos encontrada más frecuentemente en la población mundial, mientras que el situs inversus (SI) es una imagen en espejo del situs solitus, conservando la simetría de la disposición más común $n^{1,2}$.

Durante el desarrollo embrionario, en etapas tempranas el embrión guarda una simetría de ambos lados exacta, hasta alcanzar los 22 días de gestación, momento en el cual el corazón rota hacia la derecha, comenzando a presentar asimetría en la disposición

\section{Correspondencia:}

*Alejandro González-Muñoz

Cra., $18 \# 39-60$

Manizales

C.P. 110111, Bogotá, Colombia

E-mail: alegon_zalez@ hotmail.com
Fecha de recepción: 19-09-2019

Cir Cir. 2020;88(S1):79-82

Fecha de aceptación: 26-04-2020

DOI: 10.24875/CIRU.20001581
Contents available at PubMed www.cirugiaycirujanos.com (http://creativecommons.org/licenses/by-nc-nd/4.0/). 
de órganos. Este proceso es dependiente de la proteína Sonic Hedgehog (Shh), que permite la expresión de los factores de determinación derecha-izquierda, Nodal y Lefty. Cuando ocurre una secreción de la proteína Shh en el segmento derecho del embrión se genera una rotación inadecuada de las estructuras primitivas y se genera un $\mathrm{SI}^{1-3}$. El situs inversus totalis (SIT) es poco frecuente, con una prevalencia de 1 en 10.000 nacidos vivos; está presente en el 0.002 al $0.01 \%$ de la población ${ }^{3,4}$. Se desconoce la causa por la cual la proteína Shh se secreta en el lugar inadecuado, pero se ha caracterizado una tendencia del SI a encontrarse en varios miembros de una misma familia $^{2}$, lo que podría asociarse a una mutación genética sin un mecanismo claramente establecido.

EI SIT se identifica en la mayoría de los casos como un hallazgo incidental, con mayor frecuencia en adultos durante la solicitud de exámenes radiológicos por otras causas, sin encontrarse necesariamente asociado a un síndrome complejo".

La presentación de aneurismas de aorta abdominal (AAA) en pacientes con SIT es infrecuente en la literatura; se identifican pocos reportes en las bases de datos disponibles ${ }^{3-9}$. La presentación de un AAA en un paciente con SIT no necesariamente implica mayor morbimortalidad, pero su rotura se asocia a un mayor riesgo de complicaciones ${ }^{4}$. A continuación presentamos el caso de una paciente de 56 años con esta asociación.

\section{Caso clínico}

Mujer de 56 años con antecedente de hipertensión arterial compensada y SIT, que consulta al Hospital Universitario San Ignacio por un cuadro clínico de 3 días de evolución consistente en dolor abdominal de intensidad moderada y con irradiación a la región lumbar. En la exploración física de ingreso se identifica la presencia de una masa pulsátil en el mesogastrio y flanco izquierdo, sin signos de hipoperfusión distal.

Se solicita ecografía de abdomen, en la cual se evidencia un aneurisma de aorta abdominal, por lo cual se realiza una angiotomografía toracoabdominal que reporta la presencia de un aneurisma de $8 \mathrm{~cm}$ de diámetro y 10,2 cm de longitud, sin presencia de trombos en su interior ni signos de rotura, asociado a cambios imagenológicos relacionados con SIT (Fig. 1).

La paciente es llevada a cirugía para corrección del aneurisma. Se realiza laparotomía xifopúbica y evisceración de contenido intraabdominal, identificando el

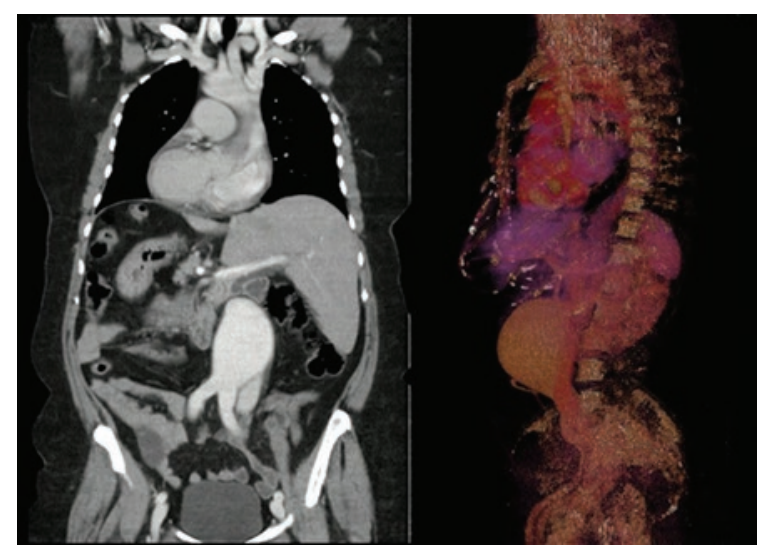

Figura 1. A: vista por tomografía computada de abdomen contrastada, con evidencia de situs inversus totalis y un gran aneurisma de aorta abdominal infrarrenal. B: reconstrucción tridimensional de angiotomografía, corte sagital, que muestra situs inversus y gran aneurisma de aorta abdominal infrarrenal.

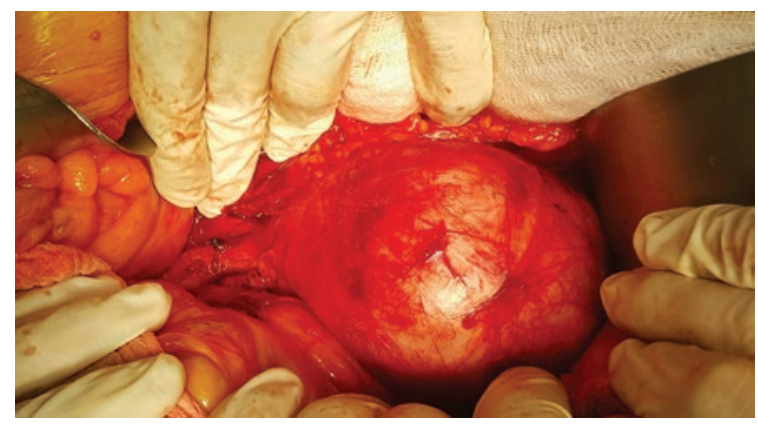

Figura 2. Identificación de aneurisma infrarrenal de aorta abdominal por laparotomía xifopúbica y posterior a la correspondiente evisceración.

cuello del aneurisma (Fig. 2). Se procede al clampeo proximal y distal del saco aneurismático, y disección del mismo, sin evidenciar trombos murales. Finalmente, se corrige el defecto con injerto de dacrón (Fig. 3), con un tiempo de isquemia del miembro inferior izquierdo de 1 hora y 26 minutos, sin presentar alteración hemodinámica ni signos de mala perfusión distal.

Durante el posoperatorio mediato, la paciente presenta ausencia de pulsos distales femoral, poplíteo y pedio de la extremidad inferior derecha, junto con palidez y disminución de la temperatura local, por lo cual se realiza Doppler arterial que muestra isquemia arterial aguda. Se decide llevar la paciente a tromboembolectomía a través de la arteria femoral de vasos suprapatelares e infrapatelares, sin que se documenten complicaciones intraoperatorias. La paciente presenta una evolución posoperatoria satisfactoria, 


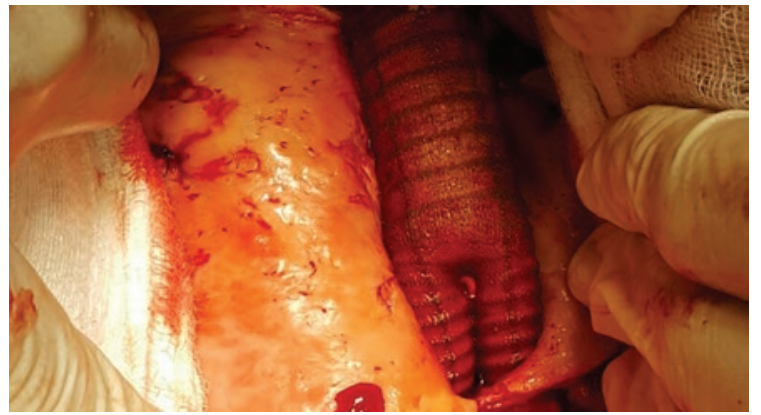

Figura 3. Imagen del injerto de dacrón en posición mediante laparotomía xifopúbica, con evisceración completa.

con resolución completa de la sintomatología, y se da de alta con signos de alarma para reconsultar.

A los 3 meses se valora en la consulta externa, donde niega la reaparición de sintomatología o la presencia de complicaciones posoperatorias.

\section{Discusión}

El SIT es una condición diagnosticada en la mayoría de los casos de forma incidental, sin encontrarse ligado a un cuadro clínico ni asociado a repercusiones que puedan poner la vida del paciente en riesgo ${ }^{1-3}$. Se debe insistir en considerar esta condición en el momento de realizar un plan quirúrgico, teniendo en cuenta los cambios en la disposición y la distribución de los diferentes órganos toracoabdominales.

La asociación entre SIT y AAA es infrecuente; hay pocos casos en la literatura, entre los cuales se han reportado pacientes con SIT y hallazgo de AAA de manera incidental, otros por cuadros clínicos benignos y algunos con rotura de $A A A^{3-9}$. En los casos reportados sin presentación de rotura del AAA, la presencia del SIT no representó mayor dificultad en la reparación quirúrgica de la malformación vascular ${ }^{3,6,9}$.

Kimura, et al. ${ }^{4}$ reportaron en 2008 el caso de una paciente de 80 años con SIT a quien se le halló un aneurisma infrarrenal de aorta, de $8.5 \times 7 \mathrm{~cm}$, quien posterior a la realización de una tomografía computarizada presentó inestabilidad hemodinámica, por lo cual fue llevada a cirugía y requirió control proximal de la aorta torácica y posterior reparo con reemplazo con injerto protésico.

En este caso, el equipo quirúrgico decidió clampar la aorta torácica descendente para lograr un control de la aorta proximal, basándose en el riesgo de que un clampeo cruzado supraceliaco transperitoneal tome mayor tiempo debido al SIT por la dificultad de ubicar las estructuras vasculares en su cambio de disposición y trayectoria. Los autores documentaron un tiempo de isquemia de 100 minutos con su intervención. Para este grupo de autores, la concomitancia de estas dos condiciones resultó un reto durante el tratamiento quirúrgico de urgencia, debido a la dificultad que representa identificar los órganos en espejo durante el sangrado intraabdominal y retroperitoneal masivo. Durante el seguimiento de esta paciente no se documentaron complicaciones relacionadas con la intervención del AAA.

Posteriormente a este caso, en 2015, Riera, et al. ${ }^{3}$ presentaron el caso de un paciente de 69 años en quien de manera incidental, durante los estudios radiológicos de una hiperplasia prostática benigna, se identificó la presencia de un AAA y de SIT. Por medio de tomografía se identificó un AAA de $6 \times 5.5 \mathrm{~cm}$ que se extendía hacia caudal hasta la bifurcación de las iliacas, pero sin mostrar evidencia de rotura. El paciente fue llevado a reparación abierta del aneurisma por laparotomía mediana, con colocación de un injerto tubular de dacrón. Como hallazgo intraoperatorio se observó el aneurisma a la derecha de la columna lumbar y la arteria mesentérica inferior saliendo a la derecha. En el seguimiento de este paciente durante 4 años no presentó complicación alguna asociada al procedimiento.

Estos autores identificaron que la concomitancia de ambas condiciones no representa un riesgo mayor para el paciente y una mayor dificultad de intervención para el cirujano al llevar estos pacientes a cirugía electiva. También indican que realizar una incisión de menor longitud y evitar la evisceración pueden asociarse a menor riesgo de íleo posoperatorio, menor estancia hospitalaria y una restauración temprana de la vía oral; evidencia también soportada por Matsumoto, et $a l .^{10}$ en una serie de pacientes, ninguno de ellos con SIT.

En 2010, Chan, et al. ${ }^{6}$ fueron los primeros en realizar una reparación endovascular de un AAA infrarrenal de $7.5 \mathrm{~cm}$, de manera electiva, en un paciente de 81 años con SIT. Este equipo quirúrgico, a través de un abordaje abierto de arterias femorales, logró realizar con éxito esta intervención, sin documentar complicaciones intraoperatorias ni posoperatorias. Estos autores consideraron que la cirugía endovascular puede presentar más beneficios al cirujano, argumentando que la reparación abierta puede presentar mayores inconvenientes por la anatomía poco familiar para el cirujano. La posición de las vísceras abdominales, especialmente el duodeno, el intestino delgado, la vena cava inferior 
y el hígado, no se asocia a limitaciones en el abordaje endovascular.

Otros autores han reportado la realización de otras intervenciones vasculares en pacientes con SIT, entre las que se encuentran reparaciones de disecciones aorticas Stanford A exitosas ${ }^{11-14}$, sin complicaciones perioperatorias. Esto concuerda con la evidencia anteriormente expuesta en cuanto a que el SIT no debería significar una mayor morbimortalidad al llevar a intervenciones de aorta a pacientes con SIT.

\section{Conclusión}

La presentación concomitante de SIT y AAA es una relación inusual, la cual durante el manejo por cirugía electiva no representa un reto terapéutico mayor respecto a un paciente con SI. La rotura de un AAA en este tipo de pacientes sí puede representar mayor dificultad para el cirujano, en comparación con la presentación normal de las estructuras toracoabdominales, lo cual se puede ver representado en un mayor tiempo para el control vascular.

La presencia de SIT debe tenerse en cuenta al intervenir quirúrgicamente a un paciente. La adecuada caracterización anatómica preoperatoria puede representar para el equipo quirúrgico un mayor dominio y planeación de la cirugía. En los reportes de esta asociación no se ha documentado ningún caso con desenlace fatal, siendo hasta ahora ocho los casos identificados, incluyendo el aquí presentado.

Como en la literatura encontrada, en nuestro caso, al no presentar rotura del aneurisma, no hubo mayor riesgo para el paciente ni más dificultad para el equipo quirúrgico. Basándonos en esto, consideramos que la intervención quirúrgica de manera electiva de estos pacientes puede realizarse como reparación abierta o de manera endovascular, lo cual queda a criterio del cirujano y de su equipo.

\section{Financiamiento}

El financiamiento estuvo a cargo de los autores.

\section{Conflicto de intereses}

Ninguno de los autores declara conflicto de intereses.

\section{Responsabilidades éticas}

Protección de personas y animales. Los autores declaran que para esta investigación no se han realizado experimentos en seres humanos ni en animales.

Confidencialidad de los datos. Los autores declaran que han seguido los protocolos de su centro de trabajo sobre la publicación de datos de pacientes.

Derecho a la privacidad y consentimiento informado. Los autores han obtenido el consentimiento informado de los pacientes y/o sujetos referidos en el artículo. Este documento obra en poder del autor de correspondencia.

\section{Bibliografía}

1. Maldjian PD, Saric M. Approach to dextrocardia in adults: review. AJR Am J Roentgenol. 2007;188(6 Suppl):S39-49-8.

2. Spoon JM. Situs inversus totalis. Neonatal Netw. 2001;20:59-63.

3. Riera Hernandez C, Perez Ramirez P, Esteban Gracia C, Jimenez Olivera MA, Llagostera Pujol S. Situs inversus totalis and abdominal aortic aneurysm: surgical repair of an extremely uncommon association. Int $J$ Surg Case Rep. 2015;10:216-8.

4. Kimura N, Kawahito K, Ito S. Rupture of an abdominal aortic aneurysm in a patient with a situs inversus totalis: report of a case. Surg Today. 2008;38:249-52.

5. Kato T, Takagi H, Sekino S, Manabe H, Matsuno Y, Umemoto T. Abdominal aortic aneurysm in situs inversus totalis. Ann Vasc Surg. 2006;20:267-70.

6. Chan Y-C, Ting AC, Qing KX, Cheng SW. Successful endovascular infrarenal aneurysm repair in a patient with situs inversus totalis. Ann Vasc Surg. 2010;24:1134.e5-7.

7. Occhionorelli S, Navarra G, Santini M, Carcoforo P, Sartori A, Pollinzi V, et al. Symptomatic abdominal aortic aneurysm and "situs viscerum inversus." Diagnostic and therapeutic approach. J Cardiovasc Surg (Torino). 1998;39:31-3.

8. Ricci M, Deshmukh N. Situs inversus and abdominal aortic aneurysm. A case report. Vasc Surg. 1989;23:413-6.

9. Baccellieri D, Mirenda F, Mandolfino T, La Spada M, Stilo F, Spinelli F. [Symptomatic aorto-iliac aneurysm and situs viscerum inversus: case report]. Chir Ital. 2006;58:113-6.

10. Matsumoto M, Hata T, Tsushima Y, Hamanaka S, Yoshitaka H, Shinoura S, et al. Minimally invasive vascular surgery for repair of infrarenal abdominal aortic aneurysm with iliac involvement. J Vasc Surg. 2002;35:654-60.

11. Magishi K, Izumi Y, Ishikawa N, Kimura F. Stanford type A acute aortic dissection caused by blunt trauma in a patient with situs inversus. Ann Thorac Surg. 2006;81:2294-6.

12. Niino $T$, Shiono M, Inoue T, Hata M, Sezai A, Negishi N. A case of acute aortic dissection type $A$ in a patient with situs inversus. Ann Thorac Surg. 2003;75:1963-5.

13. Pecha S, Schirmer J, Yildirim Y, Kolbel T, Hakmi S, Reichenspurner H, et al. Type A aortic dissection with situs inversus. J Card Surg. 2013;28:747-8.

14. Sato M, Nagaya K, Hatakeyama M, Komatsu T. Aortic arch replacement in an elderly patient with dextrocardia and situs inversus. Eur $\mathrm{J}$ Cardiothorac Surg. 2013;43:e28. 